\title{
Philosophiques
}

\section{Le rêve matérialiste, ou « Faire par la pensée ce que la matière fait parfois "}

\section{Charles T. Wolfe}

Volume 34, numéro 2, automne 2007

URI : https://id.erudit.org/iderudit/016993ar

DOI : https://doi.org/10.7202/016993ar

Aller au sommaire du numéro

Éditeur(s)

Société de philosophie du Québec

ISSN

0316-2923 (imprimé)

1492-1391 (numérique)

Découvrir la revue

Citer cet article

Wolfe, C. T. (2007). Le rêve matérialiste, ou « Faire par la pensée ce que la matière fait parfois ». Philosophiques, 34(2), 317-328.

https://doi.org/10.7202/016993ar
Résumé de l'article

Cet article vise à expliciter la notion de "rêve matérialiste " à partir d'une réflexion sur l'ouvrage de Diderot, Le Rêve de D'Alembert. Quel lien y a-t-il entre le matérialisme philosophique proclamé dans ce livre et la forme du rêve qui donne un caractère inédit à la présentation de cette philosophie? Une approche purement textuelle montrerait, déjà, une indissociabilité particulière entre forme et contenu; mais l'approche proposée ici s'attache à la manière dont une certaine idée du rêve sert de condition nécessaire conceptuelle à l'élaboration du matérialisme de Diderot : à l'affirmation de l'unicité du monde matériel sans perdre de vue l'état mental d'un sujet rêvant. 


\title{
Le rêve matérialiste, ou « Faire par la pensée ce que la matière fait parfois $» "$
}

\author{
CHARLES T. WOLFE \\ Unit for History and Philosophy of Science, University of Sydney \\ c.wolfe@usyd.edu.au
}

\begin{abstract}
RÉSUMÉ. - Cet article vise à expliciter la notion de « rêve matérialiste » à partir d'une réflexion sur l'ouvrage de Diderot, Le Rêve de D'Alembert. Quel lien y a-t-il entre le matérialisme philosophique proclamé dans ce livre et la forme du rêve qui donne un caractère inédit à la présentation de cette philosophie? Une approche purement textuelle montrerait, déjà, une indissociabilité particulière entre forme et contenu; mais l'approche proposée ici s'attache à la manière dont une certaine idée du rêve sert de condition nécessaire conceptuelle à l'élaboration du matérialisme de Diderot : à l'affirmation de l'unicité du monde matériel sans perdre de vue l'état mental d'un sujet rêvant.
\end{abstract}

\begin{abstract}
This article seeks to explicate the notion of what I term the 'materialist dream', on the basis of an examination of Diderot's work D'Alembert's Dream. What might the connection be, between the materialist philosophy put forth as the content of the work, and the form of the dream which gives a highly idiosyncratic character to the presentation of this philosophy? A purely textual, literary analysis would itself show a particular kind of inseparability between form and content here; but the analysis suggested here focuses on how a certain idea of the dream serves as a necessary conceptual condition for the elaboration of Diderot's materialism : for the affirmation of the unity of the material world without losing sight of the mental states of a dreaming subject.
\end{abstract}

C'est un état bien singulier que celui du rêve. Aucun philosophe que je connaisse n'a encore assigné la vraie différence de la veille et du rêve'.

Quand la philosophie se trouve dans une impasse dont la science ne peut pas la sortir, face à une limite ou un «dehors ", il lui reste une botte secrète, une sortie cachée : le rêve. Pourtant, elle doit également se garder de ne pas fonder sa réflexion sur une rêverie, car elle tomberait alors dans la sphère du privé même et n'aurait aucune force de conviction sur laquelle s'appuyer quand elle chercherait à convaincre un interlocuteur. Certes, depuis Locke, nous connaissons

* Cette réflexion sur le lien entre matérialisme et rêve au sein du Rêve de D’Alembert de Diderot doit beaucoup aux analyses de Alain Gigandet sur Diderot et Lucrèce, et de Jean-Claude Bourdin sur la forme du Rêve, ainsi qu'aux travaux du groupe de recherches auquel j'ai appartenu sur le Rêve au sein du CERPHI (ENS LSH, 2000-2005), dirigé par Jean-Claude Bourdin, Colas Duflo, et Annie Ibrahim, travaux qui ont abouti à la publication de l'Encyclopédie du Rêve de D’Alembert, Paris, Éditions du CNRS, 2006.

1. Diderot, "Promenade Vernet ", Salon de 1767, CEuvres complètes, d. Dieckmann-ProustVarloot, Paris, Hermann, 1975- (cité DPV), vol. XVI, p. 230. 


\section{Philosophiques / Automne 2007}

le rôle des expériences de pensée dans la construction d'un argument philosophique, mais celles-ci servent principalement une fin déflationniste de réduire l'argument d'autrui à des bases absurdes. On imagine mal un Socrate rêvant: "Sais-tu, mon cher Ménon, qu'hier j'ai rêvé d'un esclave capable de résoudre les plus vieux problèmes de la géométrie?".

Un projet systématique se porterait mal s'il était fondé sur un rêve; mais une philosophie spéculative et éclectique - au sens pluraliste et non fondationnaliste, donc asystématique donné à ce mot dans l'article "ÉCLECTISME " de l'Encyclopédie de Diderot et D'Alembert ${ }^{2}$ — qui souhaitera pousser plus loin la réflexion sans rattacher chaque idée neuve à une chaîne de vérités préexistante, pourra les exprimer par un rêve. Mais justement, ce que j'appellerai ici le rêve proprement matérialiste n'est pas un pur artifice littéraire, un emballage pour un contenu théorique totalement distinct de sa forme. Dans ce sens, un "rêve " possède une dimension corporelle vécue que n'a pas un "songe ", ce que l'histoire de ces expressions semble confirmer: pendant longtemps, "songer» est associé à " penser », alors que « rêver» est associé à « délirer » ${ }^{3}$. Ainsi, la notion que cet article vise à expliciter, celle de "rêve matérialiste ", est tout le contraire des " songes » ou "rêveries » philosophiques; ceux-ci sont soit des artifices visant à présenter un ensemble d'idées déterminées - les mythes dans la République de Platon, ou justement le Songe de Platon de Voltaire - soit des prolongations de l'espace conceptuel du moi, chez Descartes ou Rousseau.

Je pense que c'est dans ce sens qu'il faut interpréter la phrase assez mystérieuse de l'article «RÊEE» qu'on considère comme étant pour l'essentiel de Diderot (on le trouve dans l'édition de référence de ses (Euvres complètes): "L'histoire des rêves est encore assez peu connue, elle est cependant importante, non seulement en médecine, mais en métaphysique, à cause des objections des idéalistes »; que signifie cet «à cause des objections des idéalistes » ? Lisons la suite:

Nous avons en rêvant un sentiment interne de nous-mêmes, \& en même-temps un assez grand délire pour voir plusieurs choses hors de nous; nous agissons nous-mêmes voulant ou ne voulant pas; \& enfin tous les objets des rêves sont visiblement des jeux de l'imagination ${ }^{4}$.

Autrement dit, la métaphysique matérialiste a besoin du concept de rêve pour pouvoir y assimiler le « sens interne » ou «l'intentionnalité » des idéalistes, en les naturalisant, car le rêve et surtout ses objets sembleraient être des objets

2. Encyclopédie de Diderot et d'Alembert, 35 vol., Paris, Briasson, 1751-1780 ; réédition, Stuttgart/Bad Cannstatt, Frommann, 1966, V, 273a (dorénavant cité Enc., suivi du volume et de la page).

3. L'expression "songe " est plus ancienne, datant au moins du XII siècle. Voir V. C. Petrovich, Connaissance et Rêve(rie) dans le discours des Lumières, New York, Peter Lang, 1996, p. 6.

4. "REVE, s. m. (Métaphysique) : songe qu'on fait en dormant. Voyez SONGE" (Enc., XIV, 228). 
intentionnels par excellence - mais il est également un phénomène ou processus dont on peut rendre compte en termes pleinement naturalistes. L'article, très bref, se conclut avec une définition tout à fait sensualiste, qu'on retrouvera dans l'article bien plus long du médecin suisse Samuel Formey intitulé «SONGE» (auquel je reviendrai plus $\operatorname{loin}^{5}$ ): «Les choses qui nous ont le plus frappé durant le jour apparoissent à notre âme lorsqu'elle est en repos; [...] la cause des rêves est donc toute impression quelconque, forte, fréquente $\&$ dominante ( «R $\mathrm{REVE} »$, op. cit.).

Tout métaphysique qu'il est, un rêve est plus dangereux qu'un songe (seul le premier est considéré par l'Encyclopédie comme appartenant à la métaphysique) : plus matériel, plus charnel, plus terrien en ce qu'il est plus proche du délire et donc d'une causalité psychique réelle. Ce rêve inventé est alors un rêve philosophique, qui partage avec le rêve vécu la fonction de rendre pensable l'impensable, ou, pour le dire autrement, de donner une expression, une matérialité, à des idées qui seraient restées entièrement théoriques et abstraites si elles avaient été exprimées dans un « traité », un «précis », une « ontologie »... $* * *$

Dans la pensée matérialiste, il existe quelques petits rêves, tel celui de Cyrano de Bergerac, L'Autre Monde ou États et empires du Soleil et de la Lune, qui s'apparente plutôt à ce que je nommais "songe ", et un grand rêve : le dialogue en trois parties de Diderot nommé Rêve de D’Alembert ${ }^{7}$, rédigé en 1769 mais jamais publié de son vivant — ce qui montre, en passant, qu'il n'emploie pas la forme polyphonique du rêve pour «dissimuler » des idées, comme on l'a parfois $\mathrm{dit}^{8}$. L'idée générale de ce texte qui ne peut pas être exprimée tant qu'elle n'a pas pris la «consistance» du rêve est celle de la sensibilité universelle de la matière, c'est-à-dire d'une inhérence dans la matière de la sensibilité. Le caractère «vital» ou même «vitaliste» du matérialisme de Diderot, pour reprendre une expression employée d'abord par Jean Wahl, tient dans son attribution de propriétés organiques telle la sensibilité (dont la définition est reprise principalement aux avancées physiologiques de Thomas Willis et surtout Albrecht von Haller) à la matière elle-même?

5. Formey est également l'auteur d'un Essai sur le sommeil que l'on trouve dans le tome II de ses Mélanges philosophiques, Leyde, chez E. Luzac, 1754.

6. Cyrano de Bergerac, Estats et Empires de la Lune [approx. 1648] et Histoire des Estats et Empires du Soleil [1655], in CEuvres complètes, vol. 1, M. Alcover, dir., Paris, Champion, 2000.

7. Je cite le Rêve de D'Alembert dans l'édition Versini (Euvres, tome 1: Philosophie, Paris, Laffont, coll. "Bouquins », 1994), indiquée 'V' suivi du numéro de la page.

8. Pour l'analyse textuelle suivie du Rêve de D'Alembert, qui appuie, je l'espère, l'analyse conceptuelle proposée ici, je renvoie aux études de H. Dieckmann, A. Vartanian et G. May dans Diderot Studies n 17 (1973), un numéro spécial sur le Rêve, ainsi qu'à J. Chouillet , "La poétique du rêve dans les Salons ", Stanford French Review vol. 8, n 2-3 (1983).

9. J. Wahl, Tableau de la philosophie française, Paris, Gallimard, 1962, p. 54. Sur les transformations de la notion hallérienne de sensibilité chez Diderot, voir F. Duchesneau, «Diderot et la physiologie de la sensibilité ", Dix-huitième siècle, vol. 31 (1999). 
Cette attribution est présentée parfois comme une définition (l'une des propriétés de la matière serait de sentir), parfois — notamment ici dans le Rêve - comme une expérience de pensée, qui joue dans ce texte un rôle fondateur. Répondant à l'incrédulité du personnage D'Alembert, le personnage Diderot propose le célèbre Gedankenexperiment de la statue de marbre qui «s'animalise » petit à petit, devenant terre, puis chair: " par une suite d'opérations purement mécaniques, je réduirais le premier génie de la terre à une masse de chair inorganisée » (V 614). Cependant, D’Alembert éveillé — car il n'est précisément pas encore dans un état de rêve — n'en accepte pas pour autant que « la pierre sent ». La tactique employée par Diderot, à travers le personnage du dialogue qui porte son nom, est de revenir en arrière vers un état de la conscience plus primitif, non pas parce qu'il est plus simple, brut ou malléable, mais à cause de son " animisme résiduel » : il n'y pas de distinction tranchée entre le vivant et le non-vivant dans l'univers du rêve.

Cette insistance chez Diderot sur la plénitude du rêve eu égard aux limitations spatio-temporelles du monde éveillé, sa dimension onirique, vient remplacer sa fonction prémonitoire traditionnelle ${ }^{10}$; celle-ci disparaît au XVIII ${ }^{e}$ siècle, comme on le voit avec les cas de la comète ou du monstre, qui de "merveille » ou "prodige » deviendra une simple " anomalie ", là encore suivant un processus de naturalisation ${ }^{11}$. Cette plénitude, cette matérialité différencie le rêve du songe ou de la supposition théorique pure, qui n'est qu'une abstraction, celle-ci étant jugée par Diderot, ici à travers le personnage du médecin Bordeu, comme « un signe vide d'idée » (V 667). De plus, on peut considérer que l'état de rêve, dans lequel les fonctions polarisantes de l'état de veille (telles que la concentration, la focalisation, etc.) sont éteintes, est le microcosme qui correspond, au niveau macrocosmique, à cette sensibilité universelle de la matière. Le sujet rêvant est (comme) la matière sensible, dans un état de réceptivité pure; la rêverie est présentée ici comme un abandon des frontières du moi ( « je suis un peloton de points sensibles [...] tout presse sur moi et je presse sur tout ", V 638).

Le rêve, c'est donc du concret. Chaque fois que Diderot en invente un (d'abord ceux de Mangogul et Mirzoza dans Les Bijoux indiscrets, vingt ans avant le Rêve de D'Alembert), il le fait afin de construire une situation dans laquelle le "temple des systématiciens ", au "royaume des hypothèses ", sera détruit, ici par le jeune géant Expérience. Cependant, cette destruction a bien lieu dans le rêve, mais elle produit sa fin. Ce premier rêve de Diderot n'est pas un opérateur du "plus » de réalité, à la différence du fonctionnement « fictif » du rêve proprement matérialiste, qui permet de libérer un personnage des contraintes (physiques, perceptives) du monde réel, du monde dans lequel

10. Diderot écarte la fonction prémonitoire du rêve dans Les bijoux indiscrets, chap. «Les songes ", DPV III, p. 182.

11. Voir A. Ibrahim, "Le statut des anomalies dans la philosophie de Diderot ", Dixhuitième siècle, vol. 15 (1983), et Ibrahim, dir., Qu'est-ce qu'un monstre?, Paris, PUF, 2005 ; C.T. Wolfe, dir., Monsters and Philosophy, Londres, Kings College Publications, 2005. 
nous vivons, afin d'exprimer les «suppositions » de la philosophie spéculative. Car, ne l'oublions pas, le rêve matérialiste doit faire progresser la philosophie, en tant qu'elle est à la recherche d'ordres multiples, de liaisons, de symétries au-delà de l'ordre du visible; ainsi, face à l'invisibilité de l'ordre interne du vivant, elle doit «rêver» les divers concepts-hypothèses proto-scientifiques: les «molécules» ou unités minimales du vivant, les polypes ou êtres «mitoyens » entre les règnes dont la propriété la plus frappante est de se régénérer, les générations spontanées et l'épigenèse de l'embryon qui signifient tous deux aux yeux de Diderot l'indécidabilité et le dynamisme interne de la matière vivante...

Ce rôle expérimental du rêve le rapproche de l'activité de l' 'interprète de la nature ", cette figure baconienne qui est présentée dans l'ouvrage de Diderot Pensées sur l'interprétation de la nature (1753). L'interprète de la nature peut affirmer par «conjectures » la cohérence de l'univers, y compris sa clôture causale, avant ou sans que la science expérimentale soit en mesure de la confirmer. D'ailleurs, Diderot associe lui-même l'activité cognitive nommée "conjecture ", c'est-à-dire mettre en rapport spéculatif différents éléments perçus, aux "rêves d'un malade ", à la divination. Le flux d'idées dans une conversation à plusieurs voix, leur(s) mécanisme(s) d'association, sont comme ceux des rêves : "Les rêves d'un malade en délire ne sont pas plus hétéroclites [mais cependant] il n'y a rien de décousu ni dans la tête d'un homme qui rêve, ni dans celle d'un fou ${ }^{12}$. » Dans le rêve matérialiste, on ne vérifie et on ne falsifie pas des propositions, mais on accumule des faits, toujours plus de faits ! Toutefois ces faits ne sont ni des sense-data ni des éléments constituants d'une théorie scientifique.

Le rêve n'est pas la science même; si nous avions accès aux fondements réels de la nature, à «ce qui se passe » en elle, nous n'aurions pas besoin d'analogies, de conjectures, de suppositions : de rêves. Mais inversement, et d'une manière qui rappelle tout autant l'inférentialisme humien (selon lequel «la connexion nécessaire dépend de l'inférence, au lieu que l'inférence dépende de la connexion nécessaire $\left.{ }^{13} »\right)$, toute théorie de la nature est, selon Diderot, fondée sur une analogie, comme par exemple l'analogie de la machine pour expliquer le fonctionnement du corps (après tout, même Descartes est bien obligé d'admettre, à propos du corps, qu'au mieux " on peut fort bien comparer les nerfs de la machine que je vous décris aux tuyaux des machines $\left.{ }^{14} »\right)$.

Cependant, même si l'interprète de la nature n'a pas accès à ses fondements réels et doit donc produire des conjectures dont le statut analogique les rapproche du reste des mécanismes d'association, sa réflexion est naturaliste au sens où (i) elle appartient pleinement à l'univers naturel et (ii) elle se situe

12. Lettre de Diderot à Sophie Volland, 20 octobre 1760, in Correspondance, vol. 3, G. Roth, dir., Paris, Éditions de Minuit, 1957, p. 173.

13. D. Hume, Traité de la nature humaine (1739), liv. I, ch. iii, $\mathbb{} 6$.

14. L'Homme, AT XI, 130 ; c'est moi qui souligne. 
sur un plan de continuité avec l'émergence du discours scientifique, et non pas au-dessus ou en opposition à lui. Dans ce sens, l'interprétation de la nature doit contribuer à la «théorie une» sur la «machine» de l'univers "qui est une », comme dit Diderot ; "la découverte de cette théorie est d'autant plus éloignée que la machine est plus compliquée ${ }^{15}{ }^{\prime}$. Or nos intellects ne sont pas capables de dépasser leurs limites psychologiques et spatio-temporelles. «Si nous possédions le recueil complet des phénomènes, il n'y aurait plus qu'une cause ou supposition » (ibid.). Mais nous ne possédons pas ce « recueil »; nous n'avons pas accès aux causes premières, seulement à la multiplicité des effets. Ce que Diderot appelle dans le même texte "suppléer au silence » de la nature, c'est donc nécessairement supposer et produire des conjectures, c'est rêver.

Le "grand tout» de la nature (c'est ainsi que Diderot désigne la totalité de l'univers naturel) comporte des blancs que l'histoire naturelle n'a pas remplis; elle inventera en conséquence des "cabinets d'histoire naturelle". Ceux-ci, ainsi que l'explique l'article de l'Encyclopédie qui porte ce titre (et qui est de Diderot), sont voués à combler, en tant que créations de l'esprit, les lacunes dans notre connaissance de la nature elle-même: "L'ordre d'un cabinet ne peut être celui de la nature; la nature affecte partout un désordre sublime. [...] [U]n cabinet d'histoire naturelle est fait pour instruire; c'est là que nous devons trouver en détail et par ordre, ce que l'univers nous présente en bloc ${ }^{16}$.» Produire un système de la nature, c'est inventer un arrangement particulier des «masses» ou «blocs» que la nature nous présente, une fiction, au moins à ses fondements, une supposition.

Les « suppositions » plus ou moins scientifiques de l'interprète de la nature lui permettent d'anticiper sur des découvertes scientifiques non encore réalisées, sans que son activité mentale soit une sorte de mythopoïèse spontanée, plus authentique ou première que la systématisation scientifique. Une telle distinction catégorielle n'a pas lieu d'être pour Diderot, d'une part parce qu'historiquement la constitution de la science comme ensemble théorique ordonné n'existera pas avant le XIX ${ }^{\mathrm{e}}$ siècle, mais d'autre part parce que le naturalisme de Diderot est aussi une sorte de holisme épistémologique, dans lequel un énoncé subjectif tiré de l'expérience sensorielle du sujet appartient, au même titre qu'un énoncé expérimental, au réseau total d'énoncés : la «théorie une » de la "machine [sc. de l'univers, $\mathrm{CW}]$ qui est une ».

C'est dans ce contexte théorique, et au sein d'une ontologie matérialiste, qu'il faut comprendre la rêverie du personnage de $\mathrm{M}^{\text {lle }}$ de Lespinasse, dans le Rêve. Je cite l'échange entre son personnage et celui de l'autorité, le médecin Bordeu:

15. Essai sur les règnes de Claude et de Néron, II, $\$ 97$, DPV XXV, pp. 387-389.

16. "Cabinet d'histoire naturelle ", in Diderot, Euvres, DPV VI, p. 240. On trouve des développements complémentaires dans les articles «Animal» et "Encyclopédie ». J'analyse ce naturalisme curieusement dépendant d'un artificialisme dans " "Cabinet d'histoire naturelle", or: the interplay of nature and artifice in Diderot's naturalism» (ms.). 
BORDEu. - Qu'est-ce qui circonscrit votre étendue réelle, la vraie sphère de votre sensibilité ?

Mademoiselle de Lespinasse. - Ma vue et mon toucher.

Bordeu . - De jour; mais la nuit, dans les ténèbres, lorsque vous rêvez surtout à quelque chose d'abstrait; le jour même, lorsque votre esprit est occupé ?

Mademoiselle de Lespinasse. - Rien. J'existe comme en un point; je cesse presque d'être matière, je ne sens que ma pensée; il n'y a plus ni lieu, ni mouvement, ni corps, ni distance, ni espace pour moi ; l'univers est anéanti pour moi, et je suis nulle pour lui.

BORDEU . - Voilà le dernier terme de la concentration de votre existence; mais sa dilatation idéale peut être sans bornes. Lorsque la vraie limite de votre sensibilité est franchie, soit en vous rapprochant, en vous condensant en vous-même, soit en vous étendant au-dehors, on ne sait plus ce que cela peut devenir.

Mademoiselle de Lespinasse. - Docteur, vous avez raison. Il m'a semblé plusieurs fois en rêve [...] que je devenais immense. [...] Que mes bras et mes jambes s'allongeaient à l'infini, que le reste de mon corps prenait un volume proportionné ; [...] que j'escaladais le ciel, ou que j'enlaçais les deux hémisphères (V 648-649).

La rêverie de cette femme qui croit quitter son corps et s'étendre aux dimensions de l'univers s'inscrit dans une philosophie matérialiste qui a montré que l'individu, l'unité du moi, était une fiction : c'est dans ce sens que le rêve matérialiste n'est pas un songe cartésien ou une rêverie rousseauiste, car il ne part pas du moi. Si je suis une infinité de parcelles de matière vivante, et que tout l'univers vit, je suis aussi tout l'univers : de même que la statue de marbre qu'imaginait Diderot au début du livre était déjà potentiellement de la terre, donc une plante, donc un animal, donc moi-même qui me nourrit de la chair de cet animal, eh bien, "depuis l'éléphant jusqu'au puceron, depuis le puceron jusqu'à la molécule sensible et vivante, l'origine de tout, pas un point dans la nature entière qui ne souffre ou qui ne jouisse » (V 637). Cela conduit à un paralogisme : je ne peux pas sentir tout l'univers ! On voit là une faiblesse potentielle de l'argument qui fonderait le matérialisme sur le rêve : au contraire, comme l'a remarqué un penseur méconnu de cette époque, le fait même qu'on puisse rêver de choses inconnues, de sensations inédites, et donc de "matières » inexistantes, devrait suffire à prouver que tout n'est pas matériel ${ }^{17}$ ! Chez Diderot, la solution tiendra à la distinction entre la «contiguité » purement spatiale et la «continuité » proprement organique : entre moi et Jupiter, il n'y a que de la contiguïté, alors qu'entre moi et le bout de mes doigts il y a de la »chair » continue. L'interaction entre différentes parties d'un organisme est possible parce que ses parties sont continues plutôt

17. C'est le raisonnement du Précis des arguments contre les matérialistes d'Isaac de Pinto, $2^{\mathrm{e}}$ édition, La Haye, P.-F. Gosse, 1775, p. 101. 
que simplement contigües; elles sont continues parce qu'elles possèdent la propriété de la sensibilité. L'unité du moi est produite par l'interaction d'une multiplicité de fibres organiques, composant le «faisceau " (V 625).

Quand un philosophe matérialiste écrit un Rêve, il ne s'agit donc pas seulement d'un savant artifice (straussien) permettant à l'auteur de dissimuler son point de vue ou de le faire progresser de manière dialogique (bakhtinienne) par les influences respectives de personnage sur personnage ; sa pensée n'étant qu'un mode de la matière sensible, du grand tout, le point de vue de l'auteursujet ne peut être autre que "polypeux ", pour reprendre la formule de Jacques Proust - je rappelle le passage des lettres de Diderot au sculpteur Falconet auquel Proust fait allusion:

L'argument du philosophe n'est qu'un squelette, celui de l'orateur est un animal vivant, c'est une espèce de polype. Divisez-le et il en naîtra une quantité d'autres animaux. C'est un hydre à cent têtes. Coupez une de ces têtes, les autres continueront à s'agiter, de vivre, de menacer. L'animal terrible sera blessé, mais il ne sera pas mort ${ }^{18}$.

Le rêve matérialiste cherche à «faire par la pensée ce que nature fait quelquefois » $(\mathrm{V} 643)^{19}$; et son contenu n'est donc pas un matérialisme préconstitué « habillé » en rêve, pas plus qu'il n'est assimilable à un méta-discours fondé sur l'imagination - une pensée "poétique » qui serait soi-disant plus "profonde» qu'une pensée scientifique, comme je l'ai indiqué plus haut. Simplement, les mécanismes du rêve permettent d'aller plus loin. C'est le cas quand Bordeu, perplexe face à tant d'intertextualité, présente dès le démarrage du second dialogue $-\mathrm{M}^{\mathrm{lle}}$ de Lespinasse parle, prononce une série de phrases diverses, mais on ne sait pas si c'est réellement elle qui s'exprime ou si elle est en train de «citer» le personnage D'Alembert qui a déliré dans son sommeil, délire qui la pousse à demander conseil au médecin Bordeu —, demande : «Est-ce vous qui parlez? ", et qu'elle répond: «Non. C'est le rêveur» (V 625).

Dans le même sens, il est remarquable que lorsqu'un personnage de Diderot rencontre une difficulté conceptuelle majeure, il aime bien clore en disant: Bonsoir ${ }^{20}$, non seulement ici dans le Rêve mais également dans l'Entretien d'un père avec ses enfants ou dans la partie des Salons de 1767 connue sous le nom de la «Promenade Vernet» ( «Bonsoir mon ami » puis, quelques pages plus loin: "Adieu mon ami. Bonsoir et bonne nuit ${ }^{21}$ »). Simplement, comme le sait tout lecteur du Rêve, ici le «bonsoir » mène à un nouveau niveau de la réflexion; pas seulement de la narration, mais de l'enchầnement théorique. Quand, à la fin du premier dialogue, c'est-à-dire l'entretien entre les deux philosophes, D’Alembert dit «Adieu mon ami, bonsoir et bonne

18. Diderot, lettre XV à Falconet, septembre 1766, DPV XV, p. 145. Voir J. Proust, « Diderot et la philosophie du polype », Revue des sciences humaines, vol. 14, n 182 (avril-juin 1981).

19. Dans le texte, Bordeu l'affirme sur le mode impératif: "Faites».

20. R. Mortier, "Rhétorique et discours scientifique dans le Rêve de D'Alembert" [1976], in Le cour et la raison, Oxford, Voltaire Foundation, 1990, p. 241. 
nuit », le personnage Diderot répond: «Vous plaisantez, mais vous rêverez sur votre oreiller de cet entretien » (V 621). En d'autres termes, ce dont je n'ai pas pu vous convaincre durant notre dialogue philosophique traditionnel, vous allez finir par y croire grâce à la... matérialité du rêve et de ses associations. La parole est inséparable de la matière organique; une performance verbale et un état purement corporel sont liés par une seule et même causalité, au sein du "grand tout ».

$* * *$

Ayant décrit les grandes lignes de ce qui caractérise le rêve matérialiste, il nous reste à évacuer quelques malentendus à son sujet, en le confrontant à une objection classique, avant de conclure.

Notamment, l'idée même de rêve matérialiste ne serait-elle pas une contradiction interne, comme on le pense parfois ? Cette objection est la suivante : dans un matérialisme où tout ce qui est est réel, il ne peut pas y avoir de notion d'artifice, donc du faux, donc du rêve, sauf au sens faible où l'on peut "mesurer " le pouls, la température ou les mouvements visuels d'un sujet et dire : à l'heure H1 il s'est mis à rêver. Remarquons que ce sens «faible ", que l'on retrouve dans l'approche neurophysiologique contemporaine du rêve, "repéré trop schématiquement par la sécrétion bio-électrique du sommeil paradoxal ${ }^{22}$ ", est déjà présent à l'époque de Diderot, comme l'a montré Michel Porret ${ }^{23}$, chez le médecin suisse Samuel Formey, dans l'article «SONGE » de l'Encyclopédie. Pour Formey, le rêve est toujours lié à une sensation, et on devrait pouvoir recomposer le rêve à partir d'un complexe de sensations, en faisant l'inventaire des sensations qui affectent le dormeur. Cette approche matérialiste du rêve — qui n'est donc pas le rêve matérialiste au sens où j'emploie l'expression, pour désigner la position de Diderot — marie le mécanisme cartésien (solides, fluides) à une prise en compte de l'individualité de la personne; elle met ensemble un premier niveau qui serait celui d'un appareillage mécanique, et un deuxième niveau qui serait celui du contenu biographique. Si nous nous tournons vers l'attitude matérialiste contemporaine face au rêve, celle-ci n'y verra pas non plus grandchose de réel, ${ }^{24}$ le réduisant au processus par lequel des événements émotionnants pour un animal étant produits la veille son sommeil paradoxal augmente en conséquence de 20 à $30 \%$ dans la nuit suivante ${ }^{25}$.

21. Salon de 1767, DPV XVII, pp. 230, 237.

22. B. Cyrulnik, in D. Lecourt, dir., La pensée est-elle un produit de la sélection naturelle?, Paris, PUF, 1996, p. 32.

23. Conférence inédite au $X^{\mathrm{e}}$ Congrès international des Lumières, Dublin, juillet 1999.

24. Par exemple, D. M. Armstrong, A Materialist Theory of the Mind, Londres, Routledge Kegan Paul, 1968, pp. 303-305.

25. B. Cyrulnik, op. cit. Un autre point de vue « réductionniste» consiste à se demander, non plus comment donner une explication neurophysiologique des états oniriques, mais si ceux-ci constituent des "expériences » au même titre que l'expérience consciente. C’est ce qu'affirmera Daniel Dennett ("Are dreams experiences?", Philosophical Review, vol. 85, $\mathrm{n}^{\circ} 2$ [1976]) en réaction à la théorie "externaliste» des rêves, d'inspiration wittgensteinienne, avancée par Norman Malcolm (Dreaming, Londres, Routledge Kegan Paul, 1959) : les rêves ne sont rien d'autre que des récits inventés une fois qu'on s'est réveillé. 
Mais afin d'approcher cette grande question: comment le matérialisme peut-il penser l'artifice ?, afin de répondre à l'objection, il nous faut rapidement examiner le rapport du rêve particulier formulé par Diderot à sa forme, étant donné qu'il s'agit d'un artéfact matérialiste. L'expression de "rêve matérialiste " telle que je l'ai employée ici devrait souligner que cette forme, en l'occurrence le rêve, est irréductiblement liée au cadre matérialiste dont elle est issue.

Dans une analyse de la «métaphore comme expérience» dans le Rêve de D’Alembert, Michel Delon fait une remarque très précise et utile à notre propos:

La métaphore [...] s'articule doublement sur le projet matérialiste. L'effort pour constituer une philosophie matérialiste passe par le recensement de divers savoirs scientifiques [...] et la réflexion épistémologique, mais cherche à aller audelà de ce front des connaissances par l'intermédiaire de la fiction. Cette fiction utilise les voies de l'expérience imaginaire ou de la métaphore. Comme, par ailleurs, l'objectif d'une telle recherche est de mettre en évidence la continuité qui fait passer de la matière à la sensibilité, puis de la sensibilité à la pensée [...] et fonde ainsi le monisme, la métaphore qui établit des analogies, rapproche des champs distincts, devient une figure privilégiée. Elle appartient à la démarche matérialiste qui tendrait, en un second temps, à la réduire scientifiquement à une simple métonymie ${ }^{26}$.

Pour le dire en une phrase, par l'invention métaphorique on fonde le monisme matérialiste. Cela peut surprendre, mais souvenons-nous de la remarque du personnage Diderot à D'Alembert: "Vous rêverez sur votre oreiller de cet entretien ", c'est-à-dire que l'auteur Diderot inscrit toute expression, philosophique autant que littéraire, dans une substance unique qui serait la matière vivante - et cette substance, les sujets " limités » que nous sommes la connaissent mieux en rêve que par la raison consciente. Le Rêve de D’Alembert met en scène la possibilité que la matière puisse penser, à la fois par le refus d'une séparation ferme entre sentir et penser, mais aussi par la capacité qu'a l'esprit, par la rêverie (c'est-à-dire un processus indissociable de divagation-spéculation-supposition), de s'étendre à l'univers entier, donc à la matière elle-même. C'est le sens de l'impératif de Bordeu: "Faites par la pensée ce que nature fait quelquefois " (V 643).

Mais il faut bien voir le « second temps » dont parle Delon : une fois existant, le matérialisme tendrait à vouloir nier le rêve en tant que fiction. Le problème s'est déjà posé une fois dans l'histoire de la philosophie, et même dans l'histoire du matérialisme, avec le De rerum natura de Lucrèce, comme l'a montré Alain Gigandet ${ }^{27}$. Pas seulement parce que c'est un poème (d'autres

26. M. Delon, "La métaphore comme expérience dans le Rêve de D’Alembert ", in A. Becq et al., dir., Aspects du discours matérialiste en France autour de 1770, Caen, Université de Caen / Paris, J. Touzot, 1981, p. 290.

27. Voir son excellente analyse de Lucrèce intitulée "Matérialisme et poésie ", in J.-Cl. Bourdin, dir., Les matérialismes philosophiques, Paris, Éd. Kimé, 1997. 
auteurs, comme Nietzsche, ont eux aussi innové sur le plan formel en philosophie), mais parce qu'il cherche à exprimer par ce poème la vérité d'une philosophie, l'épicurisme, qui s'inscrit dans la lignée réductionniste de l'atomisme ancien, pour lequel les formes esthétiques n'avaient guère de sens. Nous voilà face au problème mentionné plus haut: comment concevoir l'artifice dans une pensée matérialiste?

En effet, le matérialisme, considéré comme système où tout est réel et actuel, ne pourrait pas concevoir le possible ni même le faux, au sens fort. "Avec le temps, tout ce qui est possible dans la nature, est ", dit Diderot; les espèces existantes sont celles qui n'ont pas été exterminées ${ }^{28}$. Dans un univers entièrement actuel, il ne peut pas y avoir d' "erreur ", puisqu'une erreur n'est qu'un mode déficient du réel, elle n'a pas d'existence propre ni de modalité, au sens où la logique modale étudie des propriétés possibles et non actuelles: selon la formule de Hilary Putnam, "modal properties are not part of the materialist's furniture of the world ${ }^{29}$. Et l'artifice? La notion d'artifice sous-entend une distinction entre le vrai et le faux ou le naturel et l'artificiel, qui ne semble pas permise au matérialisme, pour deux raisons, qui viennent en partie d'être indiquées: d'abord sa dimension "productiviste » ou " actualiste » : tout ce qui existe est réel et dans ce sens «vrai» (difficile dans ce cadre de donner un statut particulier à la fiction: l'Iliade, dira d'Holbach, n'est que le résultat d'un «assemblage » fortuit de molécules, de « dés pipés par la nature » ${ }^{30}$ ), ensuite son refus de critères normatifs extra-territoriaux, c'est-à-dire appartenant à une sphère qui ne serait pas celle de la nature elle-même: si un être existe, nous n'avons pas de critères avec lesquels l'évaluer autre que l'existence.

Mais si la nature de Diderot est aussi celle des cabinets d'histoire naturelle, nature monstrueuse et rêvée - malgré la continuité causale globale alors nous ne sommes plus dans un univers naturaliste au sens normatif, justement (l'attitude lucrétienne classique consistant à évaluer tous les phénomènes du point de vue de la nature, pour ensuite les réduire et éliminer ce qui n'y est pas réductible, comme par exemple la superstition), puisqu'on reconnaît que le monde tel que nous le percevons n'est qu'un «masque ${ }^{31} »$. Au contraire, ce matérialisme "moderne » (au sens où Diderot emploie l'expression polémique mais surtout programmatique de «spinosistes modernes » dans l'article

28. Diderot, Sur deux mémoires de D’Alembert, $\mathbb{1}$, Le calcul des probabilités (1761), DPV II, p. 351 ; Éléments de physiologie (inachevés, rédigés essentiellement à la fin des années 1770), DPV XVII, 295.

29. H. Putnam, "Why There isn't a Ready-Made World", in Realism and Reason: Philosophical Papers 3, Cambridge, Cambridge University Press, 1987; repris in P. Moser \& J.D. Trout, dir., Contemporary Materialism, Londres, Routledge, 1995, p. 237. Évidemment, l'univers de Diderot étant également un univers de "vicissitude » qui nie radicalement l'essence, on a pu dire à l'inverse que tout chez Diderot est modalité et contingence (J. Proust, "Diderot et la philosophie du polype ", op. cit., notamment pp. 22-24).

30. Système de la Nature (1770, $2^{\mathrm{e}}$ édition 1781), réédition, J. Boulad-Ayoub, dir., Paris, Fayard, coll. «Corpus», livre II, ch. v, note 41.

31. Diderot, Éléments de physiologie, DPV XVII, p. 296. 
"SPINOSISTE » ${ }^{32}$, pour les différencier des «spinosistes anciens ", qui sont des fondationnalistes en métaphysique) accepte la dimension poïtique de l'être humain, qui transforme le monde autour de lui. Une fois cette dimension reconnue, la différence entre la nature et l'artifice disparait. Un livre, un désir ou un mensonge sont tout aussi réels qu'une molécule ou un volcan surtout quand ils sont pensés comme modes de la matière universellement vivante et sentante (donc pensante).

Nulle réduction d'un état mental à une normalité biochimique ou plus précisément neurochimique dans le grand rêve matérialiste; au contraire, tout ce qui est "fou » et «excessif » est la condition même de la progression d'un expérimentalisme, qui ne se passe pas des conjectures, suppositions et autres constructions mentales. Faisant par la pensée ce que la matière fait parfois, le rêve matérialiste est une démultiplication des points de vue par laquelle la pensée s'étend aux dimensions de l'univers, imaginant des univers empédocléens multiples ( «Dans Jupiter ou dans Saturne des polypes humains », V 630) tout en réaffirmant la sensibilité universelle de la matière ( «Qui sait ce qu'est l'être pensant et sentant en Saturne? », V 635), au lieu d'en revenir au monologue d'un philosophe, éveillé ou somnolent.

32 Enc. XV, 477a. Pour un excellent commentaire de cet article voir Alexandre Métraux, «Über Denis Diderots Physiologisch Interpretierten Spinoza », Studia Spinozana vol. 10 (1994). 